\title{
El mérito y su impacto dentro del actual sistema de selección de magistrados federales
}

\author{
The merit and its impact on the current system \\ of selection of federal judges \\ O mérito e seu impacto no atual sistema de seleção \\ de magistrados federais
}

Le mérite et son impact dans le système actuel de sélection des magistrats fédéraux

在现行联邦地方法官选举制度中的优点及其影响

Juan R. Stinco ${ }^{1}$ Universidad de Buenos Aires, Argentina

Revista Derechos en Acción ISSN 2525-1678/ e-ISSN 2525-1686

Año 4/No 13, Primavera 2019 (21 septiembre a 20 diciembre), 91-111

DOl: https://doi.org/10.24215/25251678e332

ORCID: https://orcid.org/0000-0002-0018-8897

Recibido: 07/06/2019

Aprobado: 25/09/2019

Resumen: El presente trabajo pretende relevar la relevancia del desempeño de los postulantes para los cargos de magistrados federales en las designaciones. A tales efectos se procede a definir el "mérito" en función de los resultados obtenidos en las pruebas de oposición y la calificación de los antecedentes.

Esta indagación se relaciona con un trabajo precedente ("Las reformas del sistema de administración de justicia y los procesos de selección de magistrados", Revista Derechos en Acción, Año 4, N 12, Invierno 2019 (21 junio a 20 septiembre), 609-707, ISSN 2525-1678/ e-ISSN 2525-1686) constituyendo una profundización de la investigación.

\footnotetext{
1 Abogado (UBA), Doctor en Derecho (UBA), Magister en Derecho Administrativo y Especialista en Derecho Ambiental, Jefe de Trabajos Prácticos en la materia elementos de derecho administrativo de la Facultad de Derecho de la Universidad de Buenos Aires, Prosecretario Letrado de la Procuración General de la Nación. juanstinco@gmail.com
} 
Palabras claves: Proceso de Selección de Magistrados - Consejo de la Magistratura de la Nación - Concursos.

Abstract: This paper try to explain the importance of the evaluations that are taken to the applicants for the positions of federal magistrates. For this purpose, the "merit" is defined according to the results obtained in the opposition tests and the qualification of the background.

This inquiry is related to a previous ("Las reformas del sistema de administración de justicia y los procesos de selección de magistrados", Revista Derechos en Acción, Año 4, № 12, Invierno 2019 (21 junio a 20 septiembre), 609-707, ISSN 2525-1678/ e-ISSN 2525-1686) that constitutes a deepening of the investigation.

Keywords: selection of federal judges.

Resumo: 0 presente trabalho pretende inquirir a relevância do desempenho dos candidatos para os cargos de magistrados federais nas designações. Para esse fim o "mérito" é definido de acordo com os resultados obtidos nos testes de oposição e a qualificação dos antecedentes. Esta investigação está relacionada a um trabalho anterior ("As reformas do sistema de administração de justiça e os processos de seleção dos magistrados", Revista Derechos em Acción, Ano 4, № 12, Inverno de 2019 (21 de junho a 20 de setembro), 609- 707, ISSN 2525-1678 / e-ISSN 2525-1686), constituindo um aprofundamento da investigação.

Palavras-chave: Processo de Seleção de Magistrados - Conselho da Magistratura Nacional - Concursos

Résumé: Ce travail tente d'expliquer l'importance des évaluations des candidats dans l'attribution les postes de magistrats fédéraux. À cette fin, le «mérite» est défini en fonction des résultats obtenus dans les tests d'opposition et de la qualification des antécédents .

Ce travail de recherche est lié à un travail précédent ("Las reformas del sistema de administración de justicia y los procesos de selección de magistrados", Revista Derechos en Acción, Año 4, № 12, Invierno 2019 (21 junio a 20 septiembre), 609-707, ISSN 2525-1678/ e-ISSN 2525-1686) constituant un approfondissement de celui-ci.

Mot-clés: Procédure de sélection des magistrats, Conseil de la magistrature nationale, Concours 
摘要: 本文旨在强调申请人表现与指定中联邦地方法官职位的相关 性。为此, 根据对立测试获得的结果和背景鉴定来定义 “优点” 。("Las reformas del sistema de administración de justicia y los procesos de selección de magistrados”, Revista Derechos en Acción, Año 4, No 12, Invierno 2019 (21 junio a 20 septiembre), 609-707, ISSN 2525-1678/ e-ISSN 2525-1686)构成调查的深化

关键字: 国际行政法, 全球行政法, 全球行政, 全球行政空间, 行政法 渊源。

\section{Introducción}

Resulta pacífica la idea vinculada con que se desean a los mejores candidatos para la cobertura de los cargos y funciones públicas de relevancia. En consecuencia, tal premisa se aplica en la selección de los postulantes para los cargos de magistrados federales.

Ahora bien, resulta de particular interés trazar la correlación entre el "mérito" y la efectiva designación de un postulante para el cargo de magistrado federal.

De este modo, entendiendo al mérito como la ponderación del resultado en el examen de oposición y la valoración de los antecedentes profesionales según el reglamento del Consejo de la Magistratura vigente, indagaremos sobre cómo impactan estas variables en la efectiva selección de un postulante.

Bajo tal perspectiva, en concreto, analizaremos el impacto o relevancia efectiva de la calificación recibida en el examen de oposición y la valoración de los antecedentes profesionales que realizara el Consejo de la Magistratura -en el concurso respectivo- en la elección que el Ejecutivo realiza.

Asimismo cabe especificar las limitaciones metodológicas. En primer lugar, el "mérito" en los términos que lo hemos definido aquí, no resulta una medida de la "idoneidad" de los candidatos, aspecto que excede el marco de investigación definido más arriba. Por otro lado, la información cuantitativa que se 
presenta a continuación es de elaboración propia sobre la base de la recopilación y sistematización de información pública que puede encontrarse en la web del Poder Judicial de la Nación, sección Consejo de la Magistratura (www.pjn.gov.ar).

\section{Especificación del universo analizado}

Para desarrollar el objeto de la indagación que nos propusimos, se procedió a definir el universo a relevar. Así se constataron diversos concursos para cubrir cargos de magistrados federales que se desarrollaron bajo el régimen de selección de magistrados federales ante el Consejo de la Magistratura de la Nación actualmente vigente y que tuvieron la designación de al menos 1 magistrado entre el $1^{\circ}$ diciembre de 2015 y el $1^{\circ}$ de octubre de 2019.

De este modo, la muestra se compone de los Concursos $\mathrm{N}^{\mathrm{o}} 303,304,307,308,309,310,311,312$, 313, 314, 316, 318, 321, $322,324,325,326,327,328,335,337,344,346,375,380$ у 385, muestra que compone más del $85 \%$ del universo total.

Tal universo de 29 concursos involucra un total de 2047 postulantes inscriptos, de los cuales 532 candidatos se presentaron efectivamente a rendir el examen de oposición, y 148 de ellos compusieron las ternas, sobre las cuales se designaran 44 magistrados federales.

\section{Breve repaso por el procedimiento de selección vigente}

No es la intención profundizar en detalle el régimen de selección de magistrados federales, sino simplemente recordar sus principales instancias a efectos de comprender la información cuantitativa que en los acápites siguientes se presentará.

Así, debe comenzar indicándose que el inciso $4^{\circ}$ del artículo 99 establece que el Poder Ejecutivo "Nombra los magistrados de la Corte Suprema con acuerdo del Senado por dos tercios de sus miembros presentes, en sesión pública, convocada al efecto. Nombra los demás jueces de los tribunales federales inferiores 
en base a una propuesta vinculante en terna del Consejo de la Magistratura, con acuerdo del Senado, en sesión pública, en la que se tendrá en cuenta la idoneidad de los candidatos.".

La designación de los ministros de la Corte es una atribución que le corresponde al Presidente de la Nación con acuerdo del Senado por dos tercios de sus miembros presentes, en sesión pública convocada al efecto. Esta facultad se reglamentó mediante el Decreto $\mathrm{N}^{\circ} 222 / 03$, que establece ciertos criterios para la selección del candidato propuesto como así también un procedimiento específico.

El trámite de designación de jueces federales de primera instancia y alzadas es muy distinto. Interviene el Consejo de la Magistratura, en un procedimiento que se encuentra reglado por medio de la Resolución $\mathrm{N}^{\circ} 7 / 2014$ del propio Consejo de fecha 13 de marzo de 2014.

De este modo, el nombramiento de los demás jueces de los tribunales federales inferiores que hace el Poder Ejecutivo sobre la base de una propuesta (terna) vinculante del Consejo de la Magistratura, implica que éste último órgano colegiado elabore las ternas en función a dicho procedimiento.

Como consecuencia de dicho procedimiento por cada vacante el Consejo de la Magistratura eleva una terna que contiene 3 candidatos y, eventualmente, una lista complementaria de candidatos para completar dicha terna ante el eventual caso que uno de dichos candidatos sea designado en otra vacante.

El Poder Ejecutivo selecciona uno de los candidatos que componen la terna y eleva su pliego al Senado, para obtener el acuerdo. Dentro de esta etapa, el pliego se recibe en la Comisión Permanente Unicameral de Acuerdos, que cuenta con 17 miembros y debe dictaminar sobre los acuerdos pedidos por el Poder Ejecutivo. Para ello se entrevista al candidato ternado en una audiencia pública, y una vez que cuenta con dictamen favorable, el Senado vota su acuerdo. Con el acuerdo del Senado otorgado, posteriormente se le toma juramento ante la Corte Suprema de Justicia de la Nación. 
El procedimiento mediante el cual se integran las ternas, es seguido dentro del Consejo de la Magistratura de la Nación.

Producida la vacante, este órgano constitucional debe instar el proceso, designar jurados titulares y suplentes y realizar la convocatoria para sustanciar el concurso. Dentro del mismo, existen en la actualidad tres hitos: a) el examen escrito; b) la evaluación de los antecedentes y c) la entrevista de los postulantes que se encuentren entre los primeros puestos del orden de mérito que se confecciona con la sumatoria de los puntajes obtenidos en el examen y en la valoración de los antecedentes.

Sucintamente cabe referir que el examen escrito secreto se trata de la redacción de una sentencia, con el estilo propio del fuero e instancia que se concursa, el caso a resolver debe relacionarse con los temas y competencia del cargo concursado y cada uno de estos extremos es calificado por un jurado compuesto por docentes magistrados, docentes de facultades de derecho y docentes competencia general.

A los efectos de resguardar el anonimato se emplea un sistema de cifrado alfanumérico. La calificación puede ser de 0 a 100, y se aprueba con un mínimo de 50 puntos.

Paralelamente una subcomisión del Consejo de la Magistratura califica los antecedentes acreditados por los postulantes. Los rubros que se computan son trayectoria, especialidad, docencia, publicaciones y postgrados, cuyos puntajes máximos son 30, 40 y 10 para cada uno de los restantes rubros, respectivamente, otorgándose como máximo 100 puntos.

Cuando el jurado del examen escrito presenta las correcciones mediante un informe donde fundamenta el puntaje otorgado en cada caso. Acto seguido se realiza una audiencia dentro del Consejo de la Magistratura donde se develan las claves y mediante la fusión del informe de la calificación de los antecedentes más el puntaje obtenido en el examen de oposición se confecciona el orden de mérito provisorio, respecto del cual los concursantes pueden realizar impugnaciones, las cuales son resueltas por otra subcomisión designada especialmente a tales efectos. 
Con tales resultados, se confecciona el orden de mérito definitivo el que surge de la suma aritmética de los resultados. Luego se realiza el tercero de los hitos mencionados, que básicamente consiste en una audiencia pública ante una subcomisión del Consejo de la Magistratura donde son convocados en general hasta 6 candidatos por cada vacante. Esta instancia no tiene reglamentariamente la finalidad de acreditar conocimiento científico, sino más bien las restantes habilidades y cualidades de los candidatos en función del cargo. Con el resultado de esta instancia se confecciona el orden de mérito definitivo del cual surge la terna de tres candidatos por vacante concursada que se eleva al Poder Ejecutivo.

\section{La composición del "mérito" dentro del proceso de selección}

Conforme se indicó en el acápite anterior, el mérito de un postulante dentro del concurso respectivo aparece definido por la calificación que obtuvo en el examen de oposición al que se le agrega la puntuación de sus antecedentes profesionales. Por ello corresponde hacer una mención independiente sobre cómo operan.

\section{a. El examen de oposición}

Según prescribe la reglamentación ya citada, la prueba de oposición escrita consiste en el planteo a cada concursante de uno o más casos, reales o imaginarios, para que cada uno de ellos proyecte una resolución o sentencia, como debería hacerlo estando en ejercicio del cargo para el que se postula. Dicho examen se celebra simultáneamente y su duración no excede de 8 horas.

Asimismo debe resaltarse que los casos que se planteen deben tener una relación con los temas más representativos de la competencia del tribunal cuya vacante se concursa, y con ellos se evalúa la formación teórica como práctica de cada postulante.

También se establece que la extensión total de los casos no deberá superar las 10 págs. y deberá preverse que pueda ser resuelto razonablemente por los postulantes en el término concedido. 
Para una mejor celebración de la instancia, se prevé que el jurado designado presente un temario que indicará un conjunto de institutos procesales y de fondo sobre los que versarán los casos. Este temario es puesto en conocimiento de los candidatos.

Sobre la base de tales temarios los jurados presentan 4 casos diferentes, en sobres cerrados, de similares características, no identificables y sellados de tal modo que se garantice su inviolabilidad. Antes del comienzo del examen, se sortea cuál de dichos casos será en definitiva el que los postulantes deben resolver para la realización de la instancia de oposición.

Para ello, los concursantes utilizan la computadora y el procesador de texto provistos por el Consejo de la Magistratura, donde se prevén las especificaciones y demás procedimientos para el cumplimiento del recaudo de anonimato. Asimismo se establece que podrán utilizar únicamente los textos legales y la bibliografía y jurisprudencia mínima de consulta, que lleven consigo, siempre que se trate de una cantidad razonable.

Como anteriormente se indicara, el jurado califica las pruebas de cada concursante con hasta 100 puntos, y para ello debe considerar la consistencia jurídica de la solución propuesta dentro del marco de lo razonable, la pertinencia y el rigor de los fundamentos, y la corrección del lenguaje utilizado. En caso de no haber criterio mayoritario respecto del puntaje que merecieren todos los aspirantes o alguno de ellos, la calificación resultará del promedio de los puntajes propuestos por cada jurado.

Ahora bien, analicemos la información de la muestra relevada. Conforme indicamos, la muestra que compone nuestro universo de trabajo es de 29 concursos que involucran un total de 2047 postulantes inscriptos. De ellos sólo 532 candidatos se presentaron efectivamente a rendir el examen de oposición, por lo que este será el subconjunto sobre el cuál extraeremos información.

En el gráfico que sigue a continuación, se muestran las calificaciones máximas y mínimas, junto con el promedio general para cada uno de los concursos relevados, siendo estos datos para el total general de 58 puntos de promedio, con una calificación máxima de 98 y una mínima de 8 puntos. 


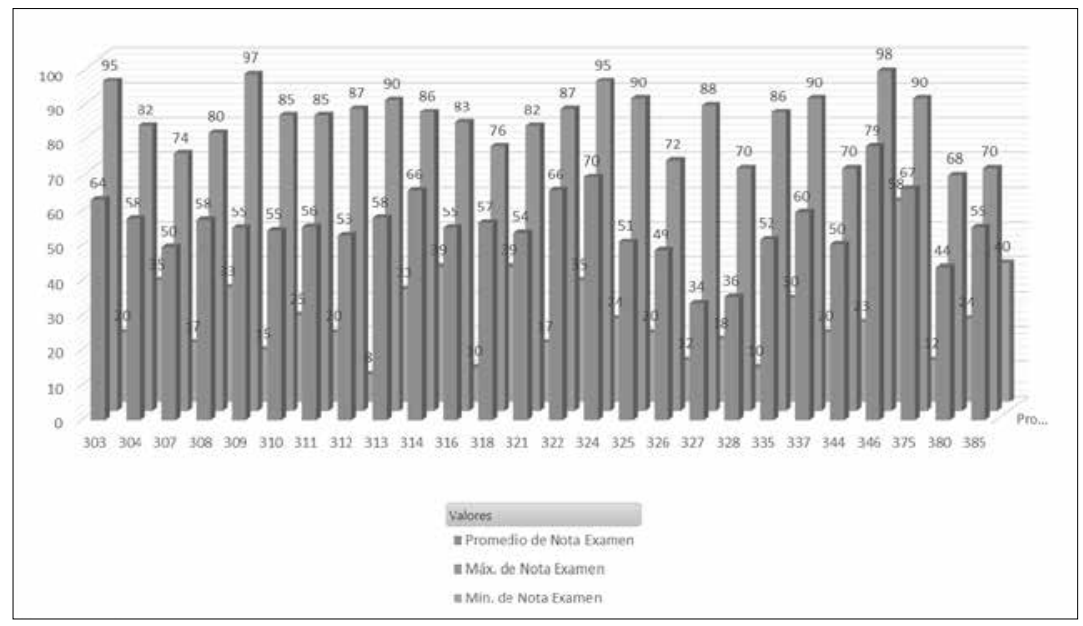

Un interrogante válido para empezar a analizar el desempeño de esta variable podría estar vinculado con la experiencia de los postulantes, considerada en función de sus años como profesional, entendidos como la cantidad de años desde la obtención de su título de abogado hasta la fecha de clausura del período de inscripción en cada concurso en cuestión. Así se obtiene el siguiente cuadro.

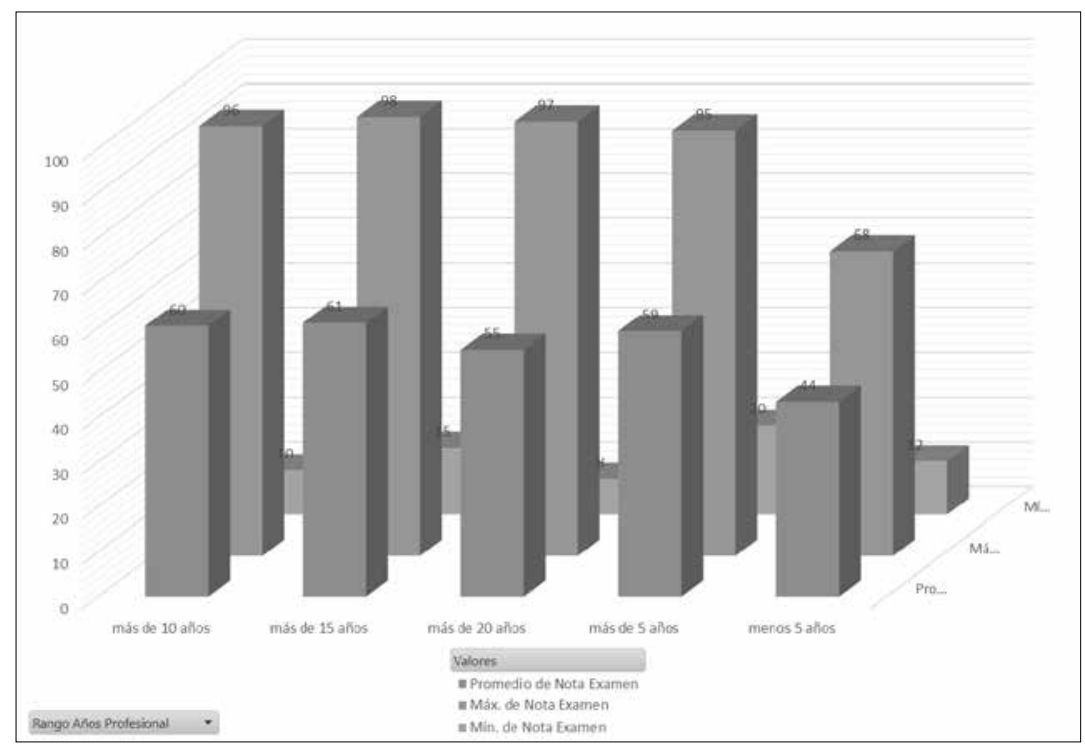


Como puede observarse, resulta un aspecto a considerar la inscripción de postulantes con menos de 5 años de haber recibido su título de grado, y en los promedios se muestra un sensible decrecimiento en las calificaciones de los postulantes con más de 20 años de experiencia profesional.

Otro aspecto que podría causar curiosidad en cómo han sido las calificaciones de los postulantes en relación con su antigüedad laboral dentro del sistema de administración de justicia, entendido en sentido amplio -comprensivo de ministerio públicos y sin importar la jurisdicción- sin clasificarlo por el cargo efectivamente desempeñado, donde el valor "NO" indica que a la fecha de la inscripción no posee antigüedad laboral alguna dentro del poder judicial.

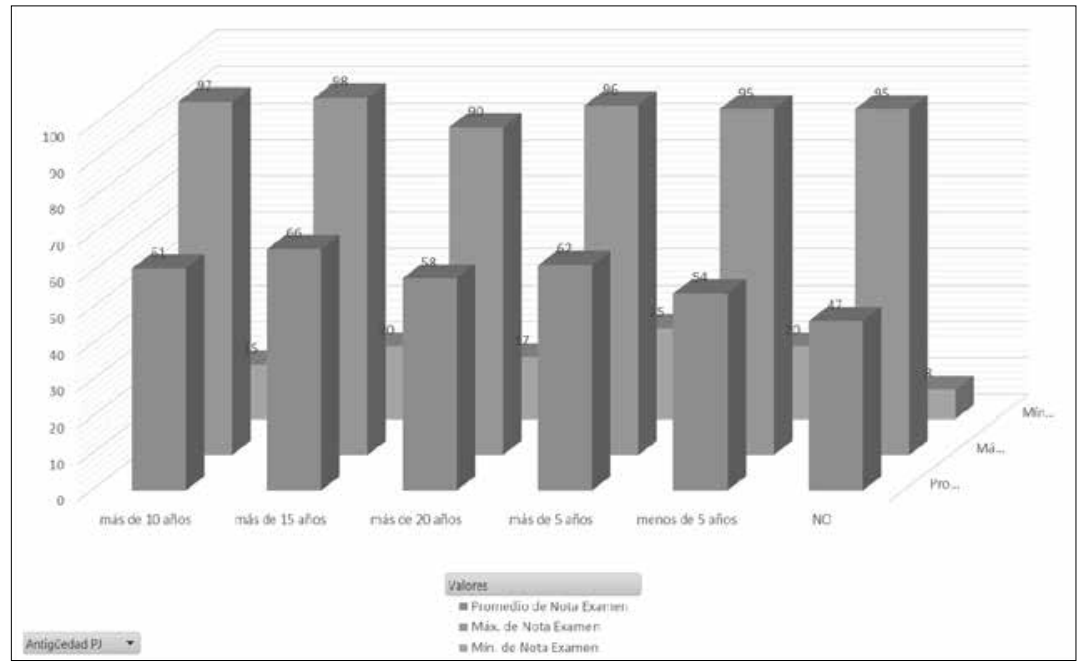

Del gráfico en cuestión claramente se observa un desempeño general promedio inferior para aquellos postulantes que no cumplieron funciones dentro del sistema de administración de justicia.

Finalmente, quizás puedan encontrarse inquietudes sobre esta variable respecto de una perspectiva de género, aspecto que puede encontrarse en el siguiente gráfico. 


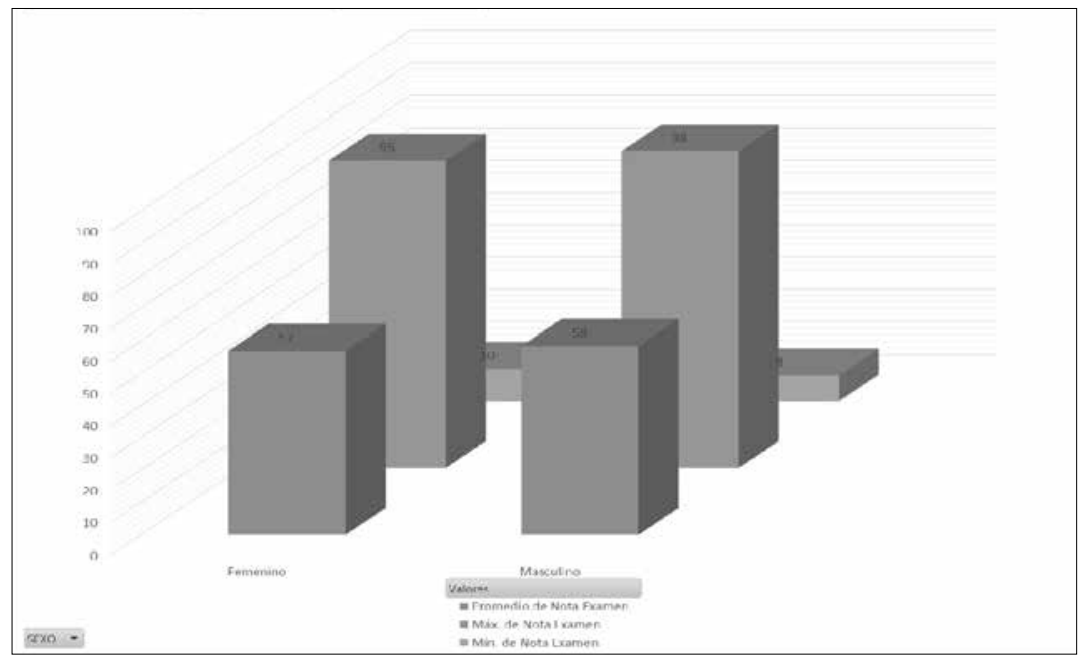

De este modo presentamos el desempeño de los 532 candidatos en el examen de oposición dentro de los procesos que componen la muestra relevada.

En el acápite siguiente realizaremos lo propio pero con respecto a la valoración de sus antecedentes profesionales.

\section{b. La calificación de los antecedentes}

En la reunión de la Comisión de Selección de Magistrados inmediatamente posterior a la realización de la prueba de oposición se sortea al consejero responsable de la evaluación de antecedentes de los inscriptos que se presentaron al examen de oposición correspondiente.

Los antecedentes de los aspirantes son calificados con un máximo de 100 puntos, considerando los antecedentes profesionales y los académicos. Los antecedentes profesionales se componen con el rubro "Trayectoria", cuyo puntaje máximo asciende a 30 y con el rubro "Especialidad", por el cual se reconocen hasta 40 puntos como máximo.

Sintéticamente señalamos que para la ponderación del rubro "Trayectoria" existe una profunda reglamentación, la que 
establece pautas discriminadas para considerar de modo diferenciado a los profesionales que se desempeñan dentro del sistema de administración de justicia, como profesionales liberales o en el mundo académico. Asimismo se establece una "Pauta Correctiva" para evitar que se pierdan puntos por parte de los postulantes que linealmente considerada su trayectoria sobrepasen el límite máximo de puntaje que pueda corresponderles. Asimismo, existe una graduación diferenciada de los valores a reconocer en función de que se trate de un concurso para cargos de primera instancia o de alzadas.

Por su parte, respecto del rubro "Especialidad", se otorgan hasta 40 puntos a los postulantes que acrediten su desempeño en funciones judiciales o labores profesionales vinculadas con la especialidad de la vacante a cubrir.

Según se indica en la reglamentación, la calificación del rubro en cuestión se ha diseñado teniendo en cuenta dos elementos: por un lado el tiempo de desempeño en cargos en el ámbito del Poder Judicial o Ministerio Público y/o en el ejercicio privado de la profesión, y/o en funciones públicas -en otros organismos, y/o la actividad académica y/o la actividad científica; y por otro, la vinculación de la especialidad de ese desempeño con la especialidad del cargo en concurso, también ponderándose si el cargo a cubrir es de primera instancia de alzada. A tales efectos, se valora el desempeño profesional en forma global a fin de determinar de modo fehaciente cuál es la especialidad que ostentan, pudiendo aplicarse un porcentual de descuento a los postulantes que acrediten una relación de modo parcial con la especialidad concursada.

De modo independiente se prevé la ponderación de los antecedentes académicos, categoría que se subdivide en "Publicaciones", "Docencia" y finalmente "Postgrados", pudiendo obtenerse por cada uno de estos rubros como máximo 10 puntos.

Para cada uno de ellos se establece una valuación detallada sobre cómo corresponde asignarse una calificación, donde cobra particular relevancia la realización de cursos ofrecidos por la Escuela Judicial del Consejo de la Magistratura. 
Analicemos cuantitativamente los puntajes asignados a los participantes que componen la muestra bajo análisis. Para comenzar podemos mostrar cómo se reparten efectivamente los puntajes totales para la muestra, a efectos de comprender cuanto termina representando en términos porcentuales cada uno de los rubros.

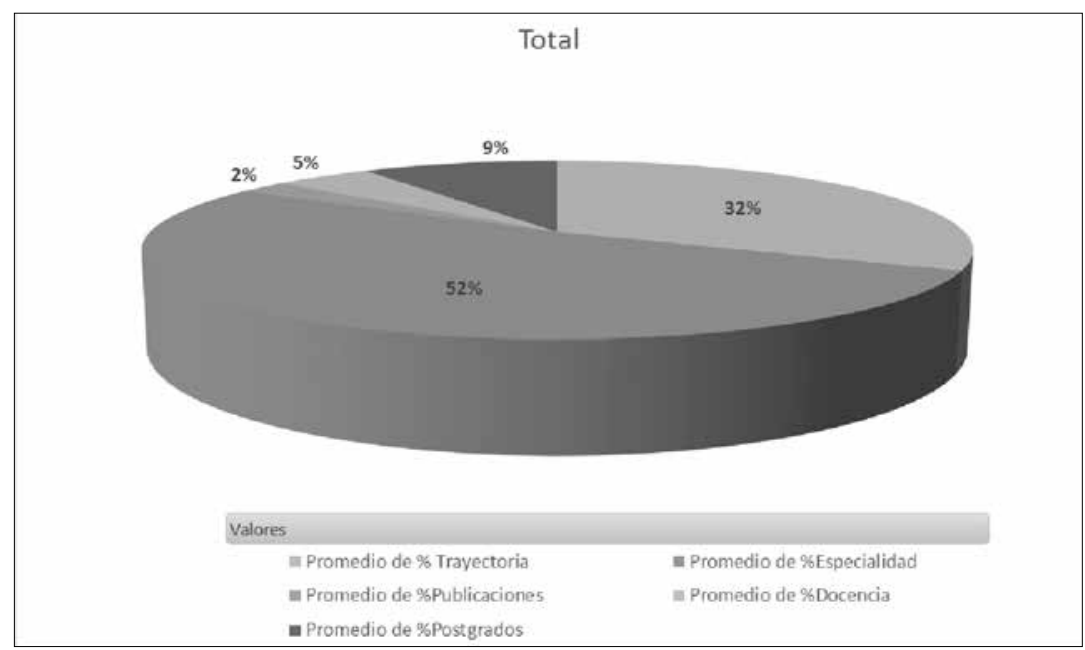

Para el caso de los magistrados designados, dichos porcentuales se reparten del siguiente modo.

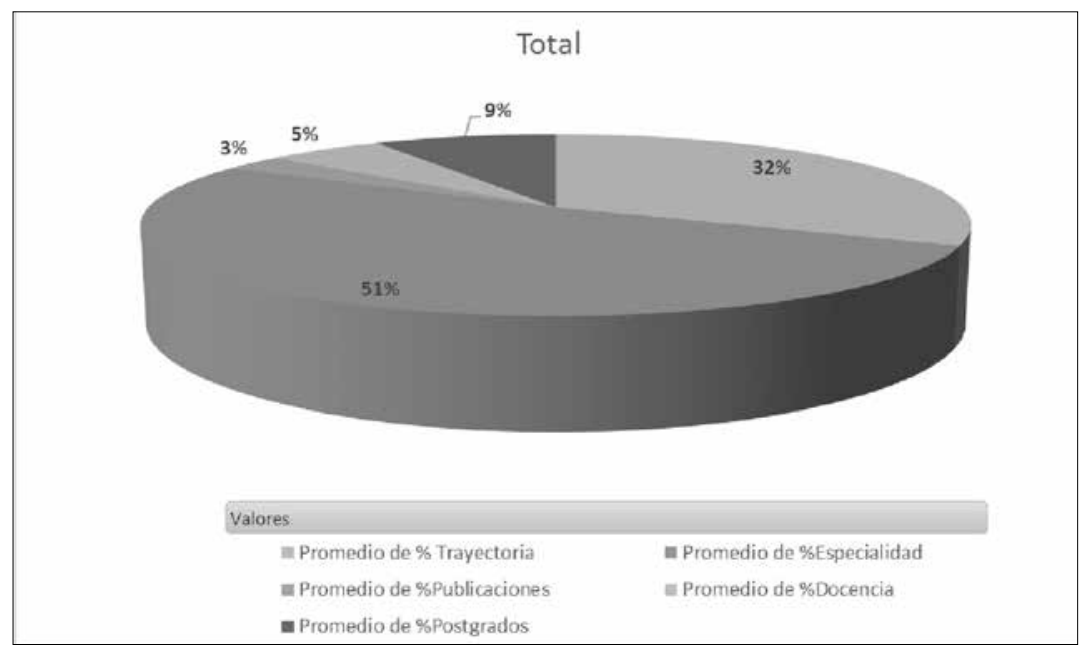


En el siguiente gráfico podemos observar la valoración de los antecedentes clasificados por Jueces designados, candidatos ternados y resto de candidatos.

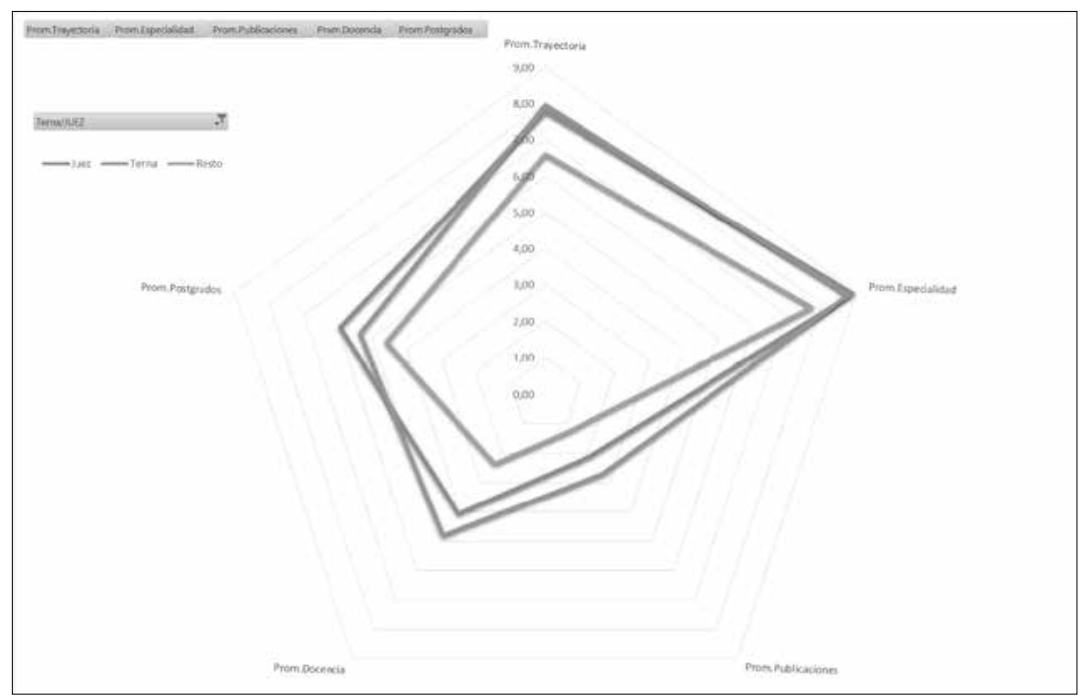

Analizando la muestra desde una perspectiva de género nos encontramos con una paridad en las calificaciones promedios, como puede observarse en el gráfico que sigue.

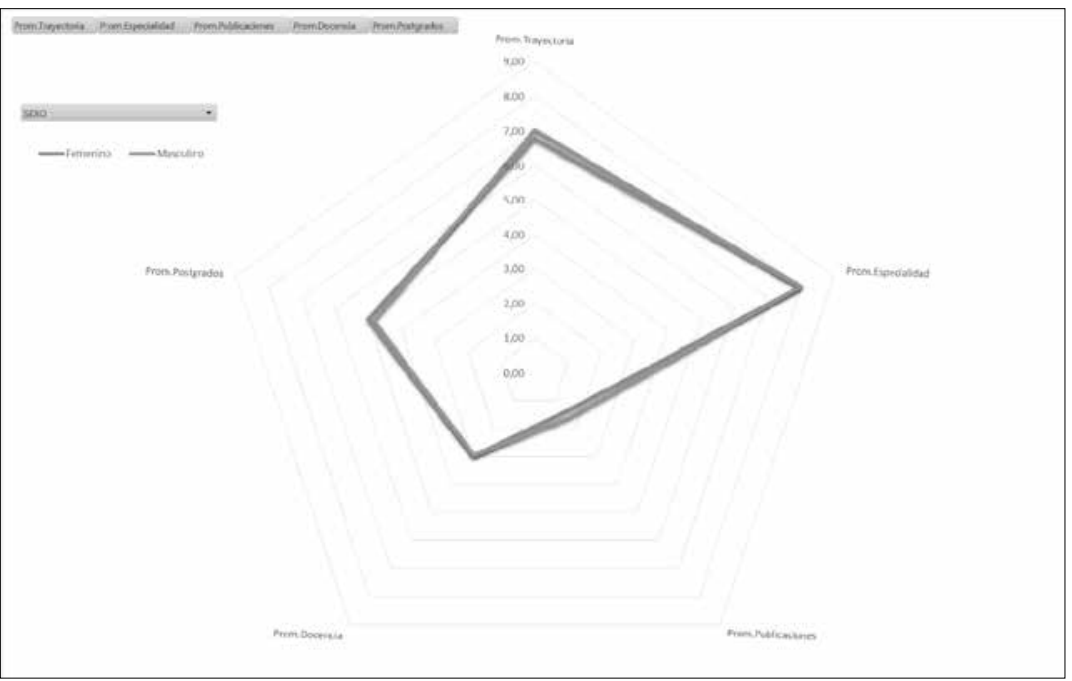


Ahora bien, si analizamos las calificaciones promedio agrupándolas en función de su desempeño dentro del sistema de administración de justicia encontramos ciertas diferencias.

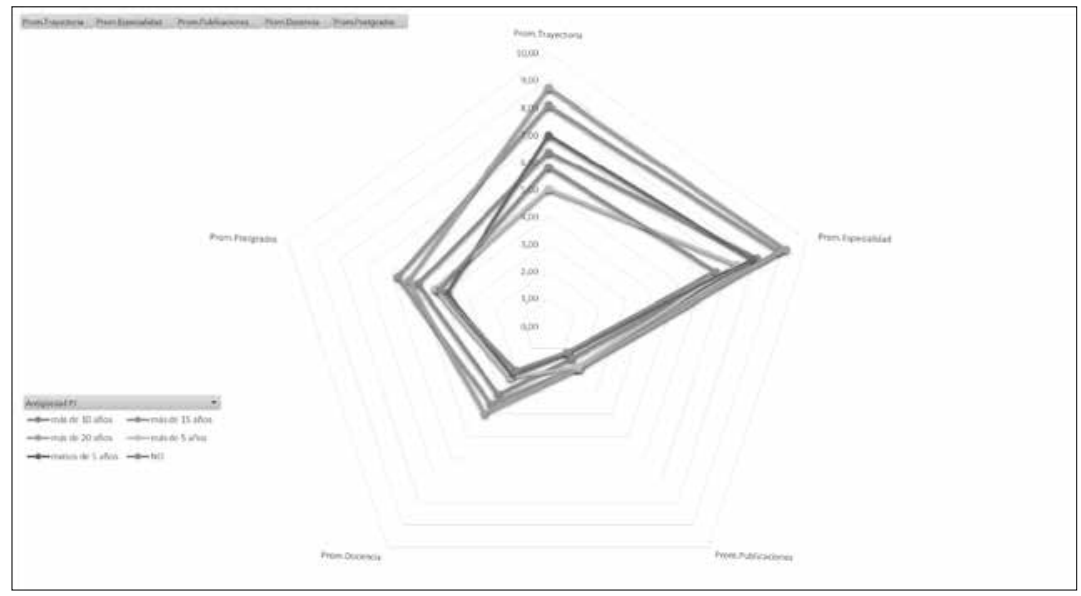

Otra forma de analizar los datos expuestos, se vincula con el cálculo de los promedios obtenidos según los rangos de años desde que los candidatos obtuvieron su título de grado.

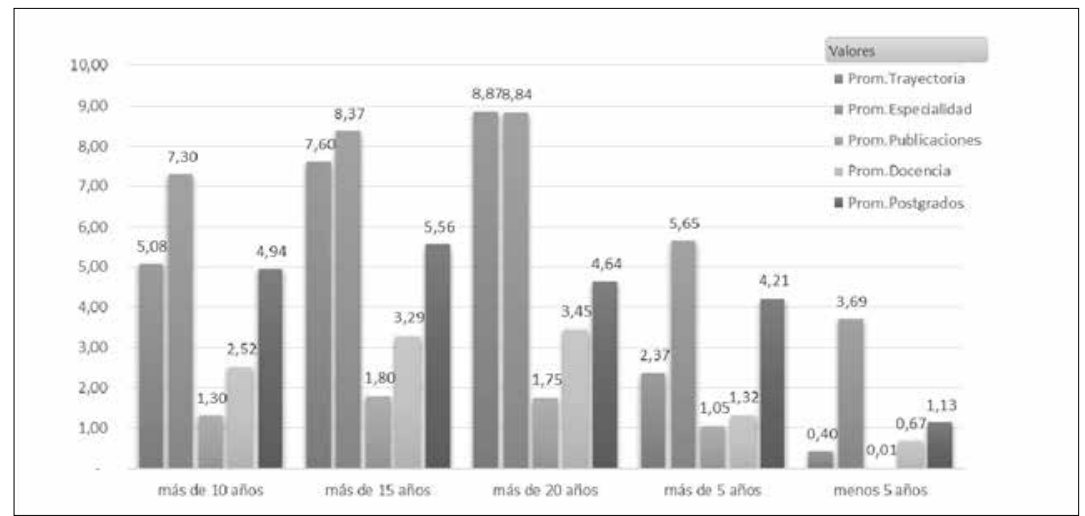

Esta disparidad justifica el análisis de una variable correctora como es la valuación de los rubros en función de rangos de años como profesionales. En el gráfico que se encuentra a continuación se realiza tal operación, pero focalizándonos en la 
variable globalmente considerada y en los rubros más representativos (Trayectoria-Especialidad).

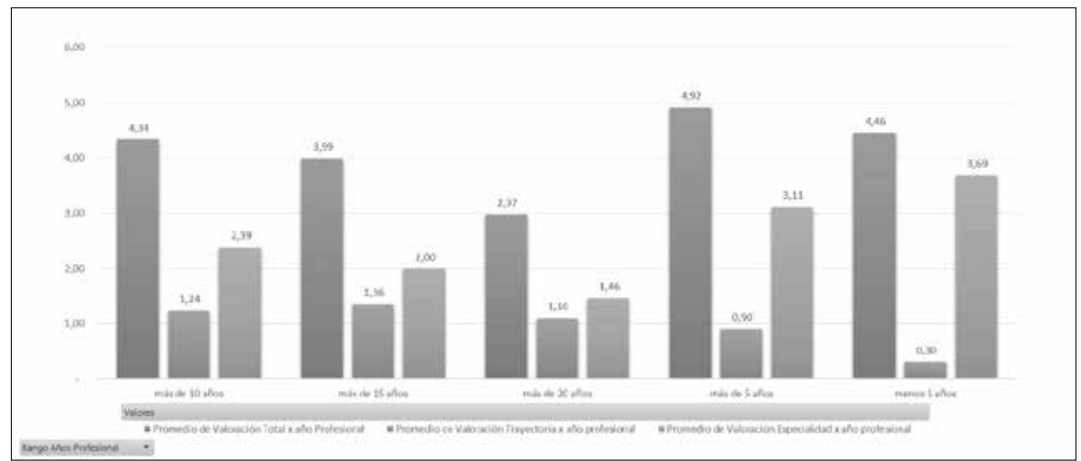

Este mismo dimensionamiento se puede realizar en función del desempeño dentro del sistema de administración de justicia, recordando que el valor "NO" es para aquellos concursantes que nunca se desempeñaron dentro del mismo.

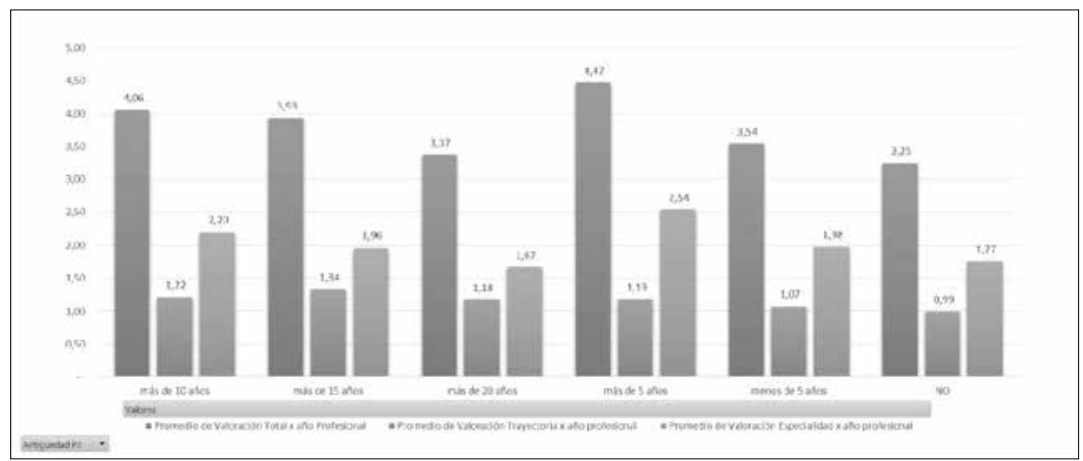

Analizado el comportamiento en los distintos rubros que se califican corresponde indagar sobre el impacto concreto en los magistrados finalmente designados. 


\section{La efectiva relevancia del "mérito" en las designaciones efectuadas}

Conforme expresamos en las primeras líneas del presente trabajo, era nuestro objetivo trazar la relevancia del "mérito" al momento de las designaciones.

A tales efectos entendimos como "mérito" el desempeño de los candidatos dentro de cada concurso en el examen de oposición y las calificaciones obtenidas en sus antecedentes.

Como primera aproximación, podemos analizar lo acontecido en la confección de las ternas, esto es los 148 postulantes que compusieron las respectivas ternas enviadas por el Consejo de la Magistratura al Poder Ejecutivo. En efecto a continuación podremos observar la representación porcentual de los candidatos ternados en función del puntaje obtenido en la calificación de sus antecedentes.

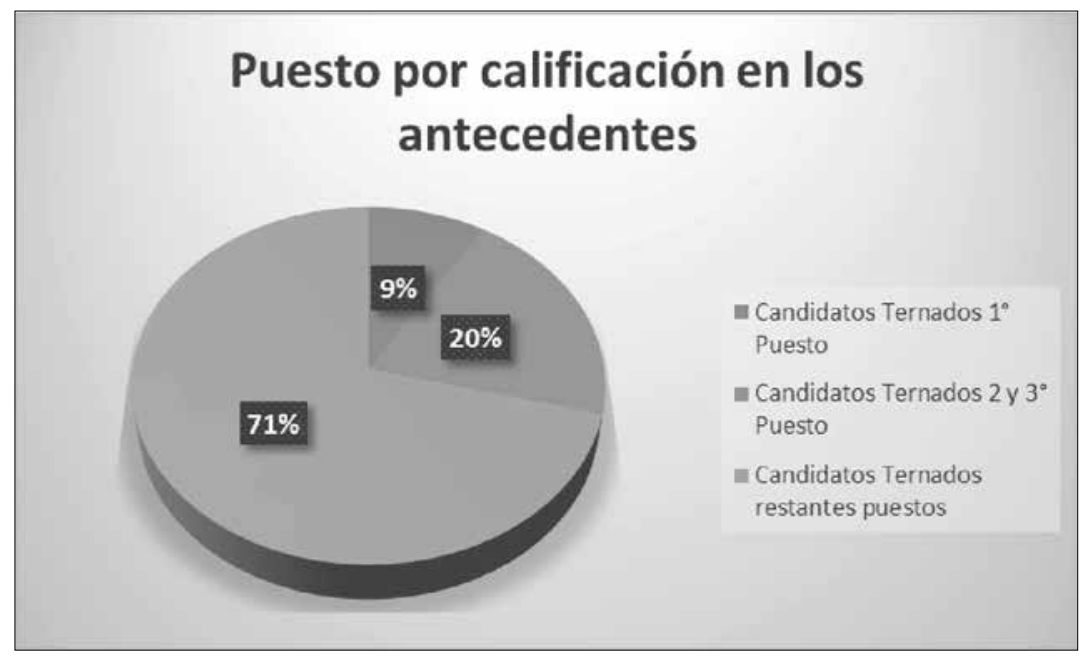

Esta misma estratificación se realiza sobre los candidatos que en definitiva fueron designados. 


\section{Puesto por calificación de Antecedentes}

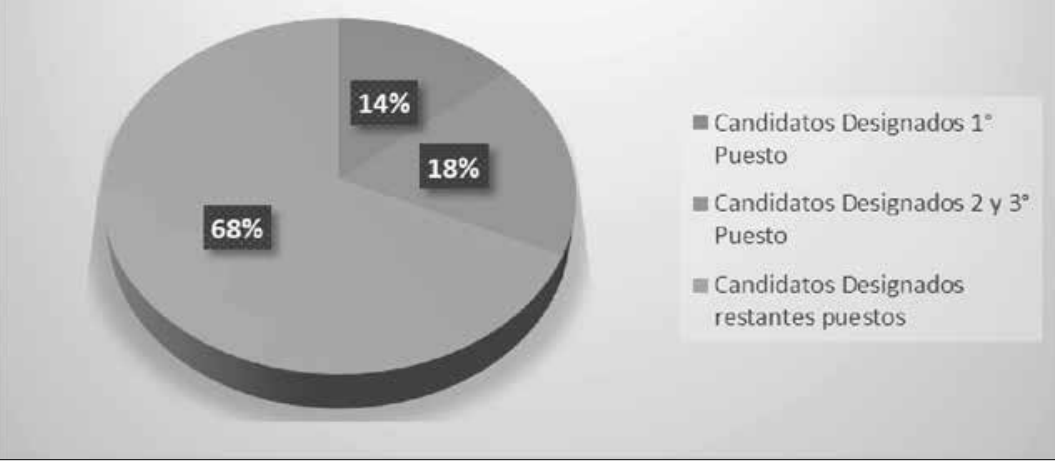

Este mismo ejercicio puede ser realizado con respecto al puesto que cada postulante ternado y efectivamente designado ocupó en la calificación asignada en el examen de oposición.

\section{Puesto por calificación en el examen de oposición}

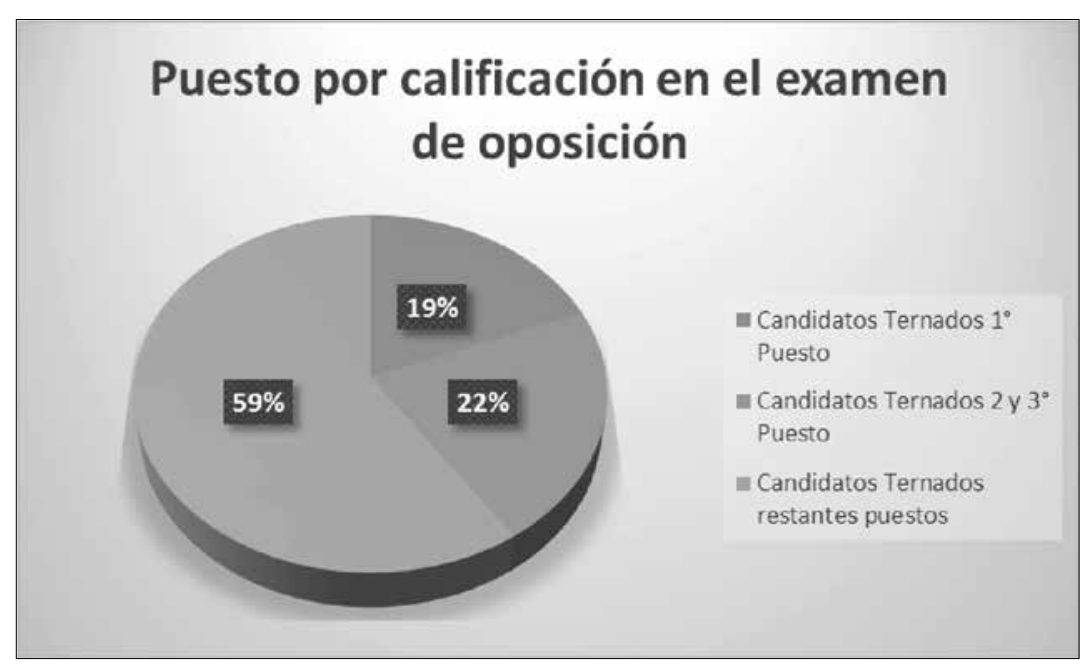

A continuación lo propio respecto de los candidatos que finalmente fueron designados. 


\section{Puesto por calificación en el examen de oposición}

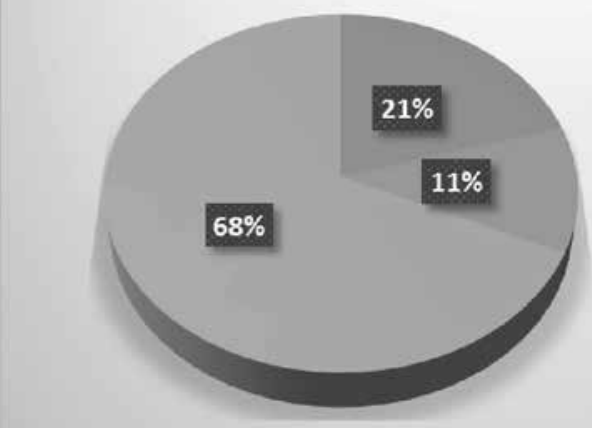

- Candidatos Designados $1^{\circ}$ Puesto

m Candidatos Designados 2 y $3^{\circ}$ Puesto

in Candidatos DEsignados restantes puestos

Ahora bien, siendo estos aspectos los centrales en el objeto de la presente indagación, procedimos a trazar la intersección entre el máximo rendimiento en el examen de oposición y la más alta calificación de los antecedentes, siempre de modo intrínseco en cada proceso concursal.

\section{Designaciones en relación al desempeño dentro del concurso}

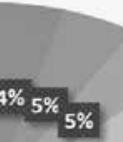


De este modo, bajo la muestra analizada, resulta que existe una baja relación del "mérito" del candidato dentro del concurso y su efectiva selección y designación, ya que tan sólo fue seleccionado un $4 \%$ de los concursantes que se destacaron con la máxima calificación en el concurso en cuestión tanto en los rubros antecedentes como en la corrección del examen de oposición.

\section{Breves corolarios preliminares}

En la intención de comenzar a comprender cómo es cuantitativamente el proceso de selección de magistrados, en otro trabajo se comenzó por relevar lo acontecido en el derecho comparado ${ }^{2}$.

Al respecto, y según analizamos en aquella oportunidad, las reformas a los sistemas de administración de justicia implementadas en Latinoamérica, han despertado el interés de investigadores, centrando sus estudios en el impacto de dichas reformas en la "independencia" misma del sistema, enfocándose en el análisis del contenido cualitativo formal, o bien en los "productos" o los resultados de su implementación.

Uno de los principales desafíos se refiere a la evaluación del impacto de la reforma en los sistemas jurídicos y judiciales; un reto que es particularmente difícil. El "producto" de un sistema legal no es fácil de cuantificar, y es igualmente difícil equilibrar la "eficiencia" contra objetivos cualitativos como "la justicia".

En la especie nos encontramos con problema estructural que afecta la casi totalidad de judicaturas de nuestra región, que consiste en la ausencia de información de lo que pasa al interior de los sistemas de administración de justicia, tanto en términos cuantitativos como cualitativos.

\footnotetext{
2 Esto fue realizado en el primero de los trabajos de investigación publicados: "Las reformas del sistema de administración de justicia y los procesos de selección de magistrados", Revista Derechos en Acción, Año 4, № 12, Invierno 2019 (21 junio a 20 septiembre), 609-707, ISSN 2525-1678/ e-ISSN 2525-1686. Puede accederse vía web en la siguiente dirección https://revistas.unlp.edu.ar/ReDeA/article/view/8757/7593
} 
Así la ausencia de información empírica imposibilita cualquier esfuerzo de planificación estratégica y convierte la reforma de la justicia en un catálogo de buenos propósitos sin respaldo analítico de alguna naturaleza lo que a la postre impide la comunicación con los funcionarios de otros poderes especialistas en el tema.

En esta oportunidad, se procedió a ampliar y actualizar la muestra y profundizar en la línea de indagación vinculada con el "mérito" y su ponderación en la designación efectiva.

Los resultados muestran una baja correlación entre el desempeño de los candidatos y su designación efectiva, aspecto que conforme se aclaró, no se relaciona con la acreditación de una adecuada idoneidad, sino con la relevancia de las máximas calificaciones y la elección que se realiza en la instancia de control político.

En la inteligencia de cuantificar el comportamiento de las instituciones, resultará de interés seguir la evolución de estos aspectos para contar con información certera de cara a la adecuada toma de decisiones y eventuales propuestas de correcciones. 